\title{
Video Article \\ Neuromodulation and Mitochondrial Transport: Live Imaging in Hippocampal Neurons over Long Durations
}

\author{
David B. Edelman ${ }^{* 1}$, Geoffrey C. Owens ${ }^{* 1}$, Sigeng Chen* \\ ${ }^{1}$ Department of Experimental Neurobiology, The Neurosciences Institute \\ *These authors contributed equally
}

Correspondence to: David B. Edelman at david_edelman@nsi.edu, Sigeng Chen at schen@nsi.edu

URL: https://www.jove.com/video/2599

DOI: doi:10.3791/2599

Keywords: Neuroscience, Issue 52, Mitochondria, Transport, Neuromodulation, Hippocampal neuron, Serotonin, Dopamine, Fluorescence, Timelapse, Live imaging, Stage-top incubator

Date Published: 6/17/2011

Citation: Edelman, D.B., Owens, G.C., Chen, S. Neuromodulation and Mitochondrial Transport: Live Imaging in Hippocampal Neurons over Long Durations. J. Vis. Exp. (52), e2599, doi:10.3791/2599 (2011).

\section{Abstract}

To understand the relationship between mitochondrial transport and neuronal function, it is critical to observe mitochondrial behavior in live cultured neurons for extended durations $s^{1-3}$. This is now possible through the use of vital dyes and fluorescent proteins with which cytoskeletal components, organelles, and other structures in living cells can be labeled and then visualized via dynamic fluorescence microscopy. For example, in embryonic chicken sympathetic neurons, mitochondrial movement was characterized using the vital dye rhodamine $123^{4}$. In another study, mitochondria were visualized in rat forebrain neurons by transfection of mitochondrially targeted eYFP. However, imaging of primary neurons over minutes, hours, or even days presents a number of issues. Foremost among these are: 1) maintenance of culture conditions such as temperature, humidity, and $\mathrm{pH}$ during long imaging sessions; 2 ) a strong, stable fluorescent signal to assure both the quality of acquired images and accurate measurement of signal intensity during image analysis; and 3) limiting exposure times during image acquisition to minimize photobleaching and avoid phototoxicity.

Here, we describe a protocol that permits the observation, visualization, and analysis of mitochondrial movement in cultured hippocampal neurons with high temporal resolution and under optimal life support conditions. We have constructed an affordable stage-top incubator that provides good temperature regulation and atmospheric gas flow, and also limits the degree of media evaporation, assuring stable $\mathrm{pH}$ and osmolarity. This incubator is connected, via inlet and outlet hoses, to a standard tissue culture incubator, which provides constant humidity levels and an atmosphere of $5-10 \% \mathrm{CO}_{2} /$ air. This design offers a cost-effective alternative to significantly more expensive microscope incubators that don't necessarily assure the viability of cells over many hours or even days. To visualize mitochondria, we infect cells with a lentivirus encoding a red fluorescent protein that is targeted to the mitochondrion. This assures a strong and persistent signal, which, in conjunction with the use of a stable xenon light source, allows us to limit exposure times during image acquisition and all but precludes photobleaching and phototoxicity. Two injection ports on the top of the stage-top incubator allow the acute administration of neurotransmitters and other reagents intended to modulate mitochondrial movement. In sum, lentivirus-mediated expression of an organelle-targeted red fluorescent protein and the combination of our stage-top incubator, a conventional inverted fluorescence microscope, CCD camera, and xenon light source allow us to acquire time-lapse images of mitochondrial transport in living neurons over longer durations than those possible in studies deploying conventional vital dyes and offthe-shelf life support systems.

\section{Video Link}

The video component of this article can be found at https://www.jove.com/video/2599/

Protocol

\section{Description of Lab-built Stage-top Incubator}

Maintaining living cells on a microscope stage for extended durations offers three major challenges: 1) ambient temperature control and regulation; 2) humidity control, i.e., maintaining moisture content of environmental atmosphere; and 3) maintenance of proper $\mathrm{pH}$ in the culture medium. These 'life support' issues are critical for experiments involving the long-term observation of cultured neurons, cells that are particularly sensitive to changes in temperature and $\mathrm{pH}$. Below, we describe a simple lab-built stage-top incubator that we designed and constructed for live imaging of neurons over extended durations. This incubator is connected, via a closed circuit, to a standard tissue culture incubator (Thermo Scientific, Asheville, NC), which provides a stable heated $\left(37^{\circ} \mathrm{C}\right)$, humidified atmosphere of $10 \% \mathrm{CO}_{2} / 90 \%$ air.

1. Incubator enclosure: The body of the incubator (Figure 1(I, II); A) was fabricated on a computerized C\&C milling machine from a solid piece of black delrin plastic. It measures $97.74 \mathrm{~mm}(\mathrm{~L}) \times 73.60 \mathrm{~mm}(\mathrm{~W}) \times 28.58 \mathrm{~mm}(\mathrm{H})$, (internal dimensions of $96.74 \mathrm{~mm} \times 72.60 \mathrm{~mm} \times 27.58 \mathrm{~mm}$ ) providing a closed volume of approximately $19,370 \mathrm{~cm}^{3}$. Two holes were drilled at either end of the enclosure and fitted with threaded brass barbs to accommodate inlet and outlet hoses from/to the tissue culture incubator. A rectangular opening measuring $70.0 \mathrm{~mm} \times 43.0 \mathrm{~mm}$ was 
milled out at the top of the enclosure to allow placement of a polycarbonate plastic window (Figure 1(I, II); B). The base of the incubator (Figure 1(I, II, III); C; top view and profile shown in detail at top left), milled from 3/16" aluminum stock, measures $159.77 \mathrm{~mm}(\mathrm{~L}) \times 109.86 \mathrm{~mm}$ (W) $\times 3.175 \mathrm{~mm}(\mathrm{D})$, and is designed to fit into the insert recess of a Leica motorized 3-plate stage (Model 11-522-068, Leica GmBh, Leipzig, Germany). Four steel posts with internal threads were attached to the corners of thebase by flush-mounted flat-head screws. These provide solid attachment points for the incubator enclosure, which has been drilled out at each corner to accept the posts (Figure 1(II, III); D). A sorbothane gasket (McMaster Carr, Inc., Elmhurst, IL) was cut and fitted to the base to provide a seal at the enclosure/base interface. Four brass thumbscrews with threading matching that of the posts are used to secure the enclosure to the base (Figure 1(II, III); D). A 35.1 mm diameter hole with a thin recessed lip was cut at the center of the incubator base to accommodate 35mm GBMs (Figure 1(III); E; detail at top left); additional bases have been designed to accommodate other culture dish sizes.

2. Heating elements: Two 10 Kohm heat sink resistors (Digi-Key Corp., Thief River Falls, MN) are affixed to the inner walls of the incubator enclosure. These are wired to a 9V DC, 500mA transformer that is plugged into an Alife $1000 \mathrm{~W}$ terrarium temperature controller (Carolina Pet Supply, Irmo, SC). A wired probe placed through a gasket in the polycarbonate window of the incubator enclosure detects internal ambient temperature and determines current flow to the resistors. In conjunction with the tissue culture incubator, the resistors provide an added measure of ambient temperature control.

3. Closed circuit atmosphere system: To provide a constant humidified and heated atmosphere of $10 \% \mathrm{CO}_{2} / 90 \%$ air, the stage-top incubator is connected, via inlet and outlet hoses (Figure 1(I); F, G), to a standard, water-jacketed tissue culture incubator (Figure 1(I); H); Forma Scientific Model 3154; Thermo Scientific, Inc., Asheville, NC). Just before connecting to the stage-top incubator, the inlet hose (Figure 1(I); F) runs into a standard aquarium pump (Figure 1(I); I; Lifegard QuietOne Model 1200, Pentair Aquatics, El Monte, CA) that has been enclosed in a sealed ABS plastic box (Figure 1(I); J; Model 1150, Pelican Products, Inc., Torrance, CA) to maintain a closed circuit. This pump promotes continuous airflow from the tissue culture incubator to the stage-top incubator. After the pump stage, the inlet hose runs to a $1500 \mathrm{ml}$ Erlenmeyer flask (Figure $1(\mathbf{I}) ; \mathbf{K}$ ) that acts as a muffler to minimize transmission of vibration from the pump to the stage-top incubator. An outlet hose (Figure 1(I); G) leading from the stage-top incubator to the tissue culture incubator (Figure 1(I); $H)$ is fitted with an enclosed computer fan (Figure 1(I); L), which, like the pump, is also intended to promote continuous airflow.

4. PFTE membrane lid for $35 \mathrm{~mm}$ GBM: Prior to placing the neuronal culture in the stage-top incubator for imaging, the $35 \mathrm{~mm}$ GBM is fitted with a special membrane lid (Figure 1(II, IV); M; detail shown at top right) to allow gas exchange and at the same time minimize evaporation of culture medium. This lid consists of a delrin plastic rim and a pre-cut sheet of PFTE membrane (Teflon; American Durafilm Co., Inc., Holliston $\mathrm{MA}$ ) that has been stretched over the rim and held in place with a viton gasket that slips into a recessed channel along the side of the delrin plastic rim.

5. Injection ports: Two holes were drilled into the polycarbonate window (Figure 1(II), center detail, yellow arrows near B) of the stage-top incubator. These were fitted with stainless steel Hamilton syringe ends to provide injection ports for administering 5-HT, DA, various receptor agonists and antagonists, and other reagents during a given experiment.

\section{Preparation of Primary Hippocampal Cultures}

All of the work is performed in either a BSL2 laminar flow hood or in a laminar flow bench. Primary hippocampal neurons are isolated from E18 rat embryos according to standard procedures ${ }^{6-7}$, and are grown in serum-free medium that has been conditioned by primary cortical astrocytes. Glial cells are prepared according to published methods ${ }^{7}$. The conditioned medium consists of low glucose DMEM, supplemented with proline $(1.76 \mathrm{ug} / \mathrm{ml})$, asparagine $(0.83 \mathrm{ug} / \mathrm{ml})$, vitamin B12 $(0.34 \mathrm{ug} / \mathrm{ml})$, glucose $(20 \mathrm{mM})$, lipid-rich BSA $(0.5 \mathrm{mg} / \mathrm{ml})$ and $2 \%$ of B27 $7^{-10}$. No antibiotics are added to the medium as they may interfere with neuronal gene transcription.

1. Cover the central glass coverslip portion of a $35 \mathrm{~mm}$ glass-bottom culture dish (GBM) with poly-D-lysine ( $0.05 \mathrm{mg} / \mathrm{ml}$ in PBS), and leave standing for two hours at $37^{\circ} \mathrm{C}$. Aspirate the poly-D-lysine solution, allow to dry for 20 minutes in a BSL2 laminar flow hood. Cover the polyD-lysine-coated coverslip portion of the GBM with laminin $(0.01 \mathrm{mg} / \mathrm{ml}$ in PBS), and leave for a minimum of 40 minutes before removing the excess laminin solution. Set aside for 20 minutes.

2. Dissect hippocampi from the brains of E18 rat embryos, and dissociate them as described in Current Protocols in Neuroscience ${ }^{6}$.

3. Dilute dissociated cells into growth medium to allow for a density of 110,000 cells per GBM. Calculate the necessary concentration of cells in your master mix according to the number of GBMs you will be preparing.

4. Place seeded GBMs in an incubator set ata temperature of $37^{\circ} \mathrm{C}$ and an atmosphere mixture of $10 \% \mathrm{CO}_{2} / 90 \%$ air.

5. Three to five days later, depending on the growing conditions of the neurons, add arabinoside $C(0.14 \mathrm{ng} / \mathrm{ml})$ to the cultures to suppress glial proliferation.

6. Allow the cultures to grow for two weeks before infecting with the lentivirus. During this time, replace one third of the volume of medium in each GBM with fresh medium every three days. Do not exceed this amount of media, as this may lead to osmotic shock and cell death.

\section{Preparation of Recombinant Lentivirus Encoding Red Fluorescent Protein}

For investigators who do not have access to facilities for producing recombinant lentiviruses, custom production by a commercial entity e.g. System Biosciences (Mountain View, CA) is an option.

A mitochondrially-targeted red fluorescent protein gene (MitoTurboRFP; Axxora LLC, San Diego, CA) is inserted into a self-inactivating recombinant feline immunodeficiency virus under the transcriptional control of the enhancer from the cytomegalovirus major immediate early gene promoter ${ }^{11}$. Recombinant lentiviruses are produced by transiently transfecting 293T cells.

1. Plate 293T cells at a density of $\sim 75,000 \mathrm{cells} / \mathrm{cm}^{2}$, the following day, co-transfect the cells with plasmids encoding the recombinant viral vector, FIV gag and pol genes, and the vesicular stomatitis virus $\mathrm{G}$ glycoprotein gene, using PolyJet (SignaGen Laboratories, Gaithersburg, $\mathrm{MD})$. A total of $12 \mathrm{ug}$ of DNA ( $4.8 \mu \mathrm{g}$ viral vector, $4.8 \mu \mathrm{g}$ gagpol plasmid, and $2.4 \mu \mathrm{g}$ VSV G plasmid) is used for every $10 \mathrm{~cm}$ culture dish of 293T cells.

2. After 24 hours, aspirate the culture medium and wash cells with phenol red-free Hanks balanced salt solution to remove residual DNA. Add $10 \mathrm{ml}$ of serum-free neural basal medium supplemented with $50 \mu \mathrm{g} / \mathrm{ml}$ lipid-rich BSA and Glutamax . 
3. The following day, harvest the supernatant, filter through a 0.2 micron low protein binding filter and add to a Vivaspin 20 polyethersulfone ultrafiltration unit (Sartorius, Concord, USA). Pre-treat the Vivaspin unit by sequentially washing with $70 \%$ ethanol, tissue culture grade water, and phosphate- buffered saline. Concentrate the virus-containing supernatant $\sim 50$-fold by centrifugation at $1500 \times \mathrm{xg}$ for 30 minutes. Aliquot the virus preparation and store in liquid $\mathrm{N}_{2}$.

4. Estimate the amount of virus by infecting rat B104 cells with an aliquot of concentrated virus, and measuring fluorescent protein expression by flow cytometry 72 hours later. The percentage of positive cells provides an estimate of the amount of virus i.e. volume of concentrated virus needed to obtain fluorescent protein expression in a given number of primary neurons.

\section{Infection of Cultured Neurons}

Hippocampal neuron cultures are infected at 14 days in vitro by simply adding the amount of virus estimated to infect up to $50 \%$ of all neurons based on flow cytometry data. No polybrene is used. Cultures are maintained for 3 days before checking for fluorescent protein expression. If the signal is too weak, then the culture is returned to the tissue culture incubator and retested after several days.

\section{General Maintenance of Closed Circuit Life Support System}

1. To maintain proper humidity, make sure that the incubator's water jacket is periodically checked and filled when necessary. Be sure to place a stainless steel or aluminum tray inside the incubator containing a small volume of water (25mm deep) and algicide.

2. When necessary, clear the inlet and outlet hoses of the incubator atmosphere circuit by emptying collected water from the catchment tubes. Periodically flush the hoses with $70 \%$ ethanol.

\section{Applying Membrane Lid to GBM and Placement in Stage-top Incubator}

1. Prior to placement of the GBM inside the stage-top incubator, replace the plastic lid with the membrane-covered lid in a tissue culture hood. Once this is done, you may move the covered GBM to the microscope.

2. Carefully seat the GBM with the membrane-covered lid in the recessed opening on the base of the stage-top incubator. To avoid jostling the GBM, make sure that the base is already in place on the microscope stage; since the base clips into the stage with some difficulty, it is best to place the GBM after the base is in position.

3. Align the incubator enclosure with the steel posts on the incubator base and lower the enclosure onto the base. Tighten the thumbscrews until a good seal is achieved between enclosure and base.

\section{Image Acquisition}

Note that the majority of available imaging software platforms have comparable functionality across a wide range of microscopes and related hardware. A variety of image acquisition and analysis software platforms are available, including MetaMorph and programs designed for specific makes of microscope, such as Leica Application Suite, Nikon's NIS-Elements, and Carl Zeiss' AxioVision. In this protocol, we describe the image acquisition and analysis procedures we performed using Slidebook 5 (Intelligent Imaging Innovations, Denver, CO). However, the constituent steps of each operation described in this protocol can be readily adapted to the use of a variety of other programs.

\section{Description of inverted fluorescence microscope, filter configurations, CCD camera, xenon light source, and imaging software:}

For dynamic imaging of mitochondrial transport in hippocampal neurons, we use a Leica DMI-6000B inverted fluorescence microscope and Model 11-522-068 motorized stage (Leica Microsystems CMS GmbH, Wetzlar, Germany) fitted with a Cooke Sensicam EQ CCD camera (The Cooke Corporation, Romulus, MI), a Sutter Lambda 10-2 filter wheel and controller (Sutter Instrument Company, Novato, CA), and a Sutter DG-4 $300 \mathrm{~W}$ xenon light source. To visualize mitochondria labeled with the MitoTurbo Red fluorescent protein, we use the combination of a 555nm filter at the DG-4 light source for excitation and filters of 600nm (peak; Sedat Quad Beamsplitter Model 86100bs fitted to Leica DM series filter cube, Chroma Technology Corp., Bellows Falls, VT) and $617 \mathrm{~nm}$ at the microscope and filter wheel, respectively, for emission.

For image acquisition and analysis, we use the digital microscopy imaging software package Slidebook 5 . This is a comprehensive package that allows fully automated control of the microscope, stage, filter wheel, camera, and light source during image acquisition, as well as a variety of image processing and analysis modules.

Below, we provide specific, step-by-step instructions for acquiring time lapse images of moving mitochondria in fluorescently labeled neurons using Slidebook 5 .

1. Make sure that the microscope, filter wheel controller, light source, and CCD camera have been turned on before opening Slidebook 5 .

2. For live imaging of mitochondria, switch to a $63 \mathrm{X}$ oil-immersion objective, lower the objective turret sufficiently to provide access to the objective below the microscope stage (and stage-top incubator), and carefully apply oil directly to the surface of the objective. Open the focus window by clicking the 'Focus Window' icon on the Slidebook toolbar. In the focus window, turn on the brightfield lamp by sliding a slider labeled 'lamp' to adjust brightfield intensity to a suitable level. Acquire focus on a field of cells and find a cell of interest.

3. Under the 'Scope' tab, select the 'CY3' fluor. Using the oculars, under fluorescent illumination find the plane of focus for mitochondria in the cells that have been labeled with the Mito TurbRed protein. Once you have acquired focus through the oculars, switch to the CCD camera and re-acquire focus if necessary.

4. Open the image capture window by clicking the 'Image Capture' icon on the Slidebook toolbar. Starting with an initial exposure time of $100 \mathrm{~ms}$ find the optimal exposure time using the 'Test' and 'Find Best' buttons; alternatively, you can use the 'Once' button. Remember that exposure time should provide ample intensity spread across the entire image field (e.g., 0-2500; indicated by histogram at the bottom of the image capture window) within a minimum duration (e.g., 200-800ms). 
5. Before starting time-lapse image acquisition, open the 'Advanced' window by clicking the 'Advanced' tab still within the image capture window. Find the 'Focus' tab, check the 'Autofous' during time-lapse/multipoint captures' option, and adjust the autofocus settings for time-lapse image acquisition. Generally, we use the following parameters for autofocus: update focus every 2-3 frames; select as the autofocus channel the fluor used for imaging labeled mitochondria, i.e., CY3; total search range 2 um for $63 x$ magnification.

6. In the 'Capture' window, under 'Capture Type' box, check the 'Timelapse' option and select the desired imaging interval and duration of imaging session under 'Timelapse Capture' options. In our studies of the effects of neuromodulators on mitochondrial transport, time-lapse imaging was performed in one hour sessions in which a total of 360 images were acquired at 10 second intervals between images.

7. Start time-lapse image acquisition by clicking the 'Start' button at the bottom, right side, of the 'Capture' window.

8. After the initial, or control, imaging session, administer neurotransmitters and other reagents via the top ports of the stage-top incubator and start a new imaging session.

\section{Image Analysis}

1. Open the image file saved after the last imaging session.

2. Individual mitochondria can be labeled in a time-lapse image series either automatically or manually by employing the masking functions provided in Slidebook 5

3. Under the 'Mask' menu, select 'Segment' from the menu bar. By sliding the low and high threshold line of the 'histogram' tool, masking all the particles in blue in the entire image, covering all the mitochondria contained in the process, but leaving particles as discrete as possible. Apply the mask to the entire image series.

4. Create a second mask (under the 'Mask' menu, select 'Create') that covers the entire image except for the process of interest in which the particles has been highlighted by the previous mask. This is accomplished, first, by using the thickest 'pencil' tool to trace the boundaries of the areas outside of the process, and then, using the 'paint bucket' tool to fill in the bounded regions. Apply this mask to the entire image series.

5. Under 'Mask,' select 'Mask Operations' and perform 'Minus' operation, i.e., the second mask is subtracted from the fist mask (the entire image except the process). A third mask will be generated in which only the process of interest is highlighted. Note that this new mask will be applied to the entire image series.

6. Under 'Annotations,' select 'Object IDs.'

7. Verify the continuity and numerical assignments of individual masked mitochondria by manually reviewing the image series (in Slidebook 5 , the 'play,' 'forward frame,' and 'reverse frame,' buttons below the image window can be used for this purpose).

8. Select the 'Mask' tab on the menu bar. Under 'Mask,' select 'Particle Tracking' and run 'Basic Particle Tracking' to generate 'Path Statistics,' including coordinates of the centroid for each masked mitochondrion.

9. Distance traveled by each mitochondrion between two adjacent image frames is calculated from the coordinates of each particle in each frame.

10. In our previous studies of the effect of neuromodulators on mitochondrial movement, we identified three distinct populations of mitochondria: 'stationary,' 'oscillatory,' and 'directionally moving.' If a mitochondrion travels less than 0.2 um (or 2 pixels, under $63 \mathrm{X}$ magnification; 1 pixel=0.10235 um) within one hour, it is characterized as 'stationary.' If a mitochondrion moves at least $0.2 \mathrm{um}$, but less than 2.5 um, in an anterograde-retrograde cycle, it is defined as 'oscillatory.' Finally, if a mitochondrion travels a net distance of more than 2.5 um in one direction, it is categorized as moving directionally. Depicted in Figure $\mathbf{2}$ is a histogram showing the movement of all labeled mitochondria in a representative experiment, as well as a pie chart showing percentage distribution of the three distinct populations of mitochondria.

11. Average speed of each mitochondrion is calculated according to total distance traveled during a given imaging session. The mean speed of a mitochondrial population is calculated by adding individual speeds and dividing by the total number of mitochondria tracked.

12. Kymographs can be generated using a module provided in Slidebook 5. Using the 'Mask' function, draw a thin line across the entire extent of a given process (e.g. axon); be sure to pass through as many mitochondrial centroids as possible. Go to the 'Mask' tab on the menu bar and select 'Advanced Operations,' and then 'Smooth Curve Analysis.' Use the default setting for smooth curve analysis or customize as desired. Run the smooth curve analysis module. At the end of the analysis, a kymograph of the imaging session will be displayed.

13. Go to the menu bar and select 'View,' and then 'Export TIFF.'

14. Open the saved .tif file containing the kymograph in Photoshop or a comparable image processing program and convert the image to inverted grayscale.

\section{Representative Results}

By tracking and analyzing mitochondrial movement in cultured hippocampal neurons, we have demonstrated a link between neuromodulation and mitochondrial trafficking. Specifically, we found that serotonin $(5-\mathrm{HT})$ or the $5-\mathrm{HT} 1 \mathrm{~A}$ receptor agonist, 8-OH-DPAT, stimulates mitochondrial movement (Figure 2A-C) ${ }^{8}$, whereas dopamine (DA) or the D2 receptor agonist, bromocriptine, inhibits mitochondrial movement (Figure 2D-G) ${ }^{9}$. 
(III

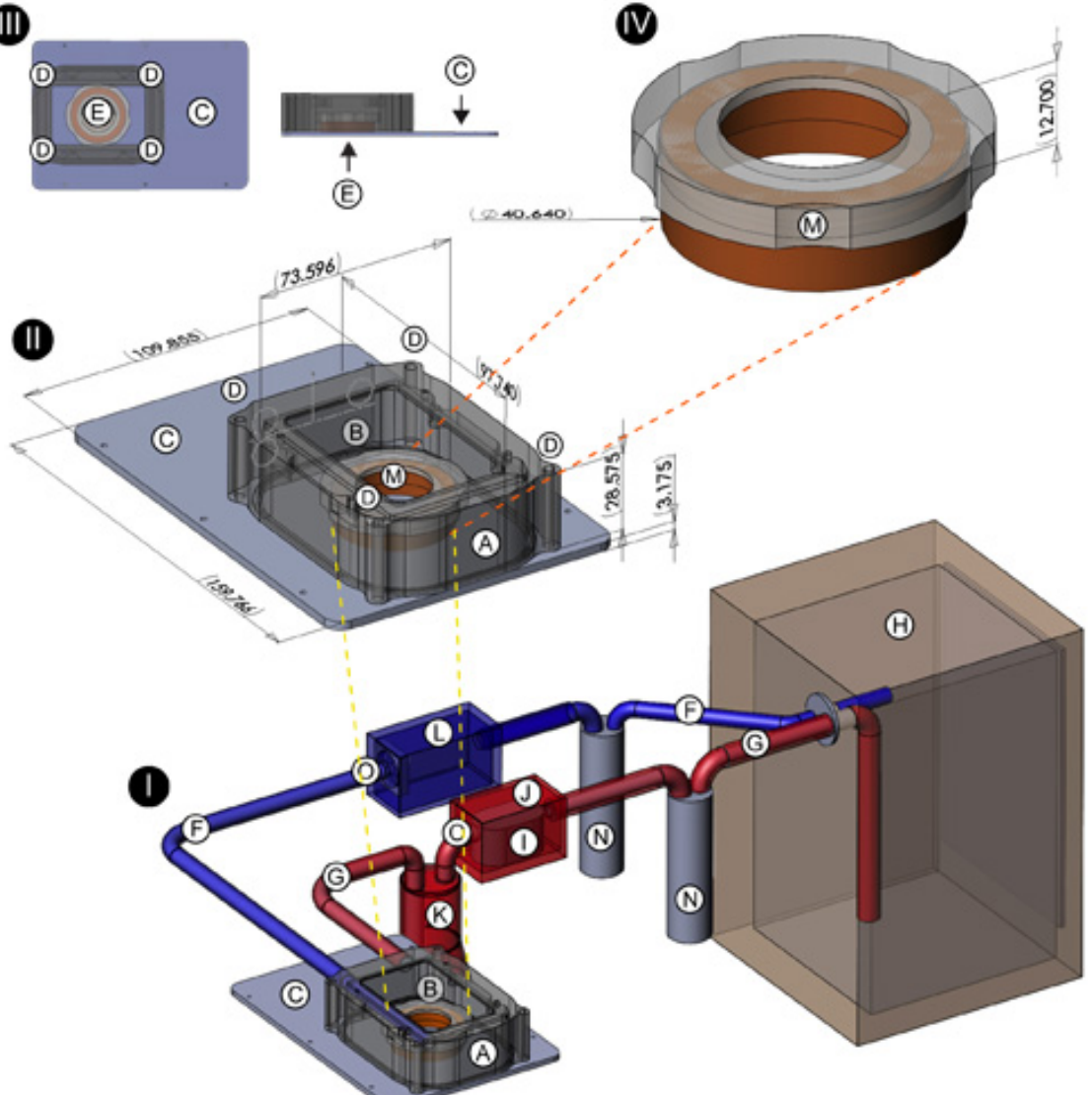

Figure 1. Design of closed circuit stage-top incubator system. The following components of the incubator system are shown: Heat-resistant delrin plastic enclosure (A); rectangular opening for polycarbonate plastic window (B); aluminum base of the incubator (C); holes to accept steel posts of base (D); $35.1 \mathrm{~mm}$ diameter hole with thin recessed lip at the center of incubator base to accommodate 35mm GBM dishes (E); nalgene hoses (connections between tissue culture and stage-top incubators; moisture traps) (F,G,N); tissue culture incubator (H); aquarium pump (I); air-tight ABS plastic box (housing for aquarium pump) $(\mathrm{J}) ; 2000 \mathrm{ml}$ Erlenmeyer flask (muffler for vibration suppression in hose before stage-top incubator) (K); plastic enclosure for cooling fan (L); plastic frame for 35mm GBM lid (M); position of plastic stopcocks $(\mathrm{O})$. Locations of ports for administration of reagents are indicated by yellow arrows in (II). 
A

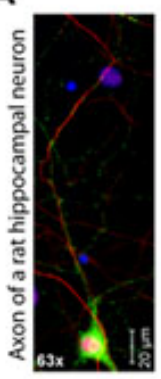

B
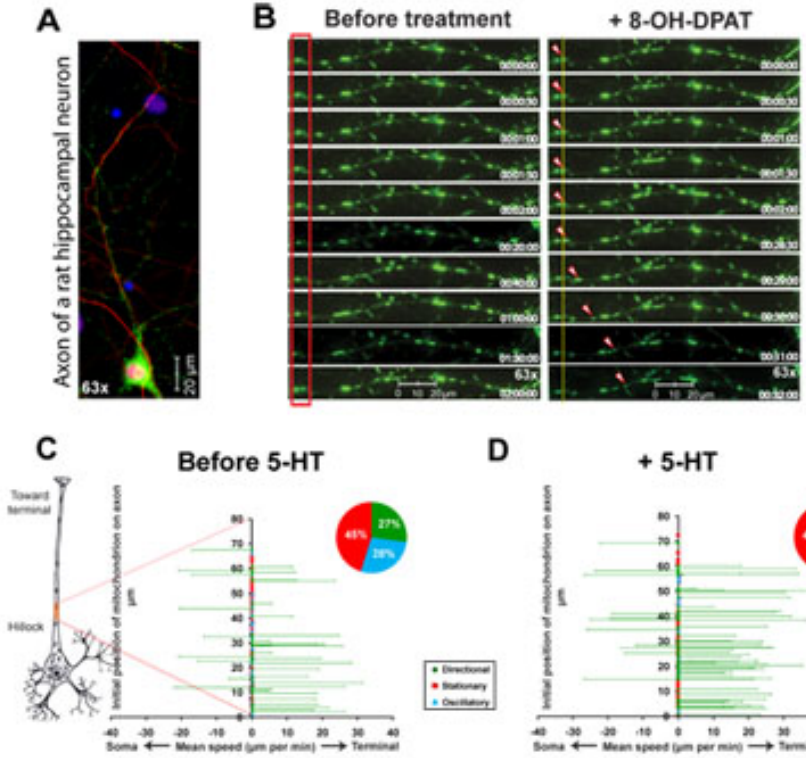

E

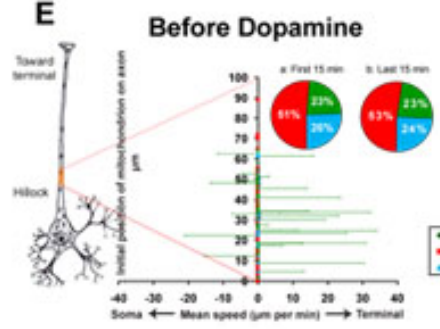

G

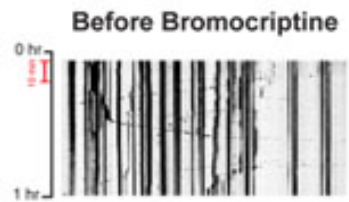

D

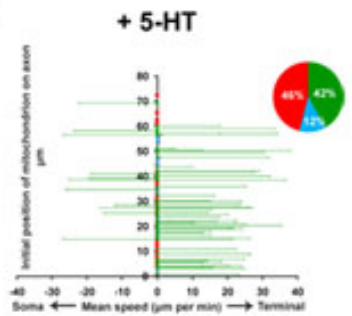

F

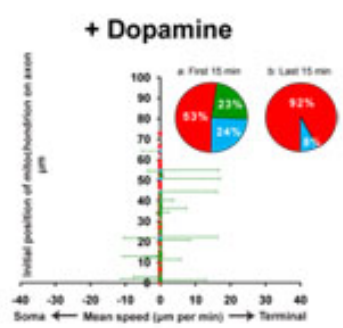

H

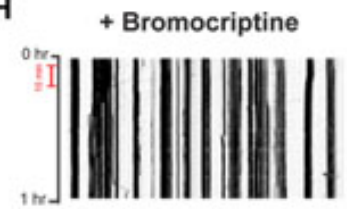

Figure 2. Representative results: regulation of mitochondrial transport. A. Axon of a typical rat hippocampal neuron in culture. Mitochondria labeled with a lentivirus-encoded fluorescent protein are shown in green; axons immunolabeled with phospho-neurofilament antibody are shown in red. Extent of axon is indicated by yellow arrowheads. Image is composed of four overlapping micrographs. B. Example of a time-lapse image series showing changes in mitochondrial movement after administration of 5-HT. Images were acquired via an inverted fluorescence microscope and stored as sequences that were later converted to Quicktime movies. A representative sequence of Images shows individual mitochondria at different time points before (left panel) and after (right panel) administration of 8-OH-DPAT, a 5-HT1A receptor agonist. Vertical red rectangle highlights a stationary mitochondrion (left) and an oscillatory mitochondrion (right) over multiple time points. The oscillatory mitochondrion indicated (left panel) is moving toward the axon terminal after treatment with 8-OH-DPAT (right panel; red-bordered white arrowheads). The vertical yellow line (right panel) indicates the starting position of the moving mitochondrion. Time intervals are shown in the lower right-hand corner of each frame. Magnification $(63 \times)$ is indicated at the lower right corner of right panel. C, D. Plots showing changes in mitochondrial movement after administration of 5-HT. Changes in mitochondrial movement before (C) and after (D) administration of 5-HT are presented as plots of velocity ( $\mathrm{X}$ axis) vs. initial positions of individual mitochondria along the axon ( $\mathrm{Y}$ axis). Velocity and proportion of stationary (red), oscillatory (blue), and directionally moving (green) mitochondria are represented in plots and pie charts (insets above plots), respectively. Red dotted lines projecting from highlighted regions of the cartoon axon to the Y-axis of each plot indicate approximate location and extent of the axon segment that was imaged. E, F. Plots showing changes in mitochondrial movement after administration of DA. Changes in mitochondrial movement before $(E)$ and after $(F)$ administration of DA are presented as plots of velocity (X axis) vs. initial positions of individual mitochondria along the axon ( $Y$ axis). Velocity and proportion of stationary (red), oscillatory (blue), and directionally moving (green) mitochondria are represented in plots and pie charts (insets above plots), respectively. Red dotted lines projecting from highlighted regions of the cartoon axon to the $\mathrm{Y}$-axis of each plot indicate approximate location and extent of the axon segment that was imaged. $\mathrm{G}, \mathrm{H}$. Representative kymographs showing mitochondrial movement in a cultured neuron before $(\mathrm{G})$ and after $(\mathrm{H})$ administration of the D1R receptor agonist, bromocriptine. The neuron was imaged for one hour before $(G)$ and one hour following $(H)$ administration of bromocriptine.

\section{Discussion}

Employing lentivirus-mediated expression of a fluorescent protein targeted to mitochondria in infected cultured neurons and an inexpensive lab-built stage-top incubator that allows imaging of live cells for extended durations, we have been able to investigate the link between mitochondrial movement and neuromodulatory signals, such as serotonin (5-HT), dopamine (DA), and acetylcholine (ACh). Our studies have helped to elucidate a signaling pathway that, for the first time, links mitochondrial trafficking to changes in the activity of neurons-modulated by 
neurotransmitters such as 5-HT and DA--that are at the heart of neural function. We find that the use of targeted fluorescent proteins permits the observation of labeled mitochondria in living cultured neurons over extended periods that may be more physiologically relevant than the much shorter durations that are possible using vital dyes. Furthermore, the intensity of the fluorescent protein signal allows us to keep exposure times short during image acquisition, minimizing the possibility of photobleaching or phototoxicity. Finally, a simple and inexpensive stagetop incubator that maintains ambient temperature, humidity, and $\mathrm{CO}_{2}$ levels, while minimizing the evaporation of media, allows us to follow mitochondrial movement in living neurons over hours or even days. Researchers who wish to fabricate a stage-top incubator for the long-term observations of mitochondria in live neurons need not follow the precise details of our design, provided that the properties of the materials used (e.g., gas permeable membrane to avoid evaporation of media) and principles applied (e.g., temperature and humidity control, buffering of $\mathrm{pH}$, maintenance of osmolarity) are generally consistent with what is described in this protocol.

\section{Disclosures}

No conflicts of interest declared.

\section{Acknowledgements}

We would like to thank Donald Hutson for contributing his technical expertise and great skill during the design and fabrication of the stage-top incubator. We are also grateful to Ayda Dashtaei for her excellent technical assistance. All work was supported by Neurosciences Research

Foundation.

\section{References}

1. Ligon, L.A., \& Steward, O. Movement of mitochondria in the axons and dendrites of cultured hippocampal neurons. J Comp Neurol 427, 340-350, doi:10.1002/1096-9861(20001120)427:3<340::AID-CNE2>3.0.CO;2-Y [pii] (2000).

2. Miller, K.E., \& Sheetz, M.P. Direct evidence for coherent low velocity axonal transport of mitochondria. J Cell Biol 173, 373-381, doi:jcb.200510097 [pii] 10.1083/jcb.200510097 (2006).

3. Macaskill, A.F., et al. Miro1 is a calcium sensor for glutamate receptor-dependent localization of mitochondria at synapses. Neuron 61, 541-555, doi:S0896-6273(09)00120-2 [pii] 10.1016/j.neuron.2009.01.030 (2009).

4. Morris, R.L., \& Hollenbeck, P.J. Axonal transport of mitochondria along microtubules and F-actin in living vertebrate neurons. J Cell Biol 131, 1315-1326 (1995).

5. Rintoul, G.L., Filiano, A.J., Brocard, J.B., Kress, G.J., \& Reynolds, I.J. Glutamate decreases mitochondrial size and movement in primary forebrain neurons. $J$ Neurosci 23, 7881-7888, doi:23/21/7881 [pii] (2003).

6. Crawley, J.N. Current protocols in neuroscience. (J. Wiley, 1999).

7. Fedoroff, S., \& Richardson, A. Protocols for neural cell culture. 3rd edn, (Humana Press, 2001).

8. Chen, S., Owens, G.C., Crossin, K.L., \& Edelman, D.B. Serotonin stimulates mitochondrial transport in hippocampal neurons. Mol Cell Neurosci 36, 472-483, doi:S1044-7431(07)00180-7 [pii] 10.1016/j.mcn.2007.08.004 (2007).

9. Chen, S., Owens, G.C., \& Edelman, D.B. Dopamine inhibits mitochondrial motility in hippocampal neurons. PLoS One 3, e2804, doi:10.1371/ journal.pone.0002804 (2008).

10. Chen, S., Owens, G.C., Makarenkova, H., \& Edelman, D.B. HDAC6 regulates mitochondrial transport in hippocampal neurons. PLoS One 5, e10848, doi:10.1371/journal.pone.0010848 (2010).

11. Curran, M.A., Kaiser, S.M., Achacoso, P.L., \& Nolan, G.P. Efficient transduction of nondividing cells by optimized feline immunodeficiency virus vectors. Mol Ther 1, 31-38, doi:10.1006/mthe.1999.0007 S1525-0016(99)90007-2 [pii] (2000). 